\title{
Paravertebral somatic nerve blocks for breast surgery in a patient with hypertrophic obstructive cardiomyopathy
}

\author{
[Les blocs paravertébraux des nerfs somatiques pour une intervention mammaire chez une \\ patiente qui présente une cardiomyopathie obstructive]
}

Chester C. Buckenmaier III MD, Susan M. Steele MD, Karen C. Nielsen MD, Stephen M. Klein MD

\begin{abstract}
Purpose: Patients with hypertrophic obstructive cardiomyopathy (HOCM), a genetic disorder resulting in idiopathic myocardial thickening, can present the anesthesiologist with significant management difficulties. This report reviews the physiology of this important disease process and describes the use of paravertebral nerve blocks (PVB) in the management of a patient with HOCM who presented for partial mastectomy with axillary lymph node dissection.
\end{abstract}

Clinical features: A 72-yr-old female presented for breast cancer surgery with a significant past medical history of HOCM diagnosed during hospitalization for non-small cell lung cancer. PVB were performed at thoracic levels $\mathrm{I}-6$ and $5 \mathrm{~mL}$ of $0.5 \%$ ropivacaine and epinephrine 1:400,000 was injected at each level. Intraoperatively the patient required no other medication for analgesia and was comfortable and conversant during the two-hour procedure. She remained pain free following the operation and did not require any opioid medication until the following day.

Conclusions: PVB provide excellent analgesia and are a useful alternative anesthetic when faced with the HOCM patient requiring major breast surgery.

\begin{abstract}
Objectif : La prise en charge anesthésique de patients atteints de cardiomyopathie obstructive (CMO), affection génétique causant une hypertrophie myocardique idiopathique, peut comporter de grandes difficultés. Le présent article passe en revue la physiologie de cet important processus morbide et décrit l'usage de blocage nerveux paravertébral (BPV) chez une patiente atteinte d'une CMO devant subir une mastectomie partielle avec évidement ganglionnaire axillaire.
\end{abstract}

Éléments cliniques : Une femme de 72 ans, devant être opérée pour un cancer du sein, avait des antécédents importants de CMO diagnostiquée pendant une hospitalisation pour cancer bronchopulmonaire "non à petites cellules". L'anesthésie a été réalisée par BPV aux niveaux thoraciques $1-6$, une injection de $5 \mathrm{~mL}$ de ropivacaine à 0,5\% et d'épinéphrine à 1:400 000 a été faite à chaque niveau. Pendant l'intervention de deux heures, la patiente n'a pas demandé d'autre analgésique, elle se sentait bien et pouvait converser. Elle n'a pas eu de douleur postopératoire et n'a eu besoin d'opioïde que le jour suivant.

Conclusion : Les BPV procurent une excellente analgésie et sont une variante efficace dans les cas de CMO nécessitant une intervention mammaire importante.

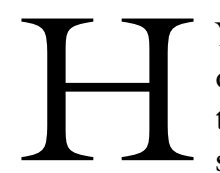
YPERTROPHIC obstructive cardiomyopathy (HOCM) is a rare, genetically transmitted disease that places patients at significant risk of sudden cardiac death. ${ }^{1}$ Patients with HOCM develop an idiopathic myocardial thickening that usually predominates in the interventricular septum. The enlarged septum can result in left ventricular outflow obstruction leading to angina pectoris, syncope, tachydysrhythmias, and congestive heart failure. ${ }^{2}$ Though morbidity and mortality during normal activity is low, HOCM patients have a higher incidence of congestive heart failure and other cardiac events when placed under surgical stress. ${ }^{3}$

Data describing the successful anesthesia management of HOCM in noncardiac surgery are limited. A recent Medline search (keywords: hypertrophic obstructive cardiomyopathy, anesthesia) revealed 33 articles that described the anesthetic management of HOCM in adults since 1966. Of these 33, the majority are in Japanese journals and none of these reports

From the Department of Anesthesiology, Duke University Medical Center, Durham, North Carolina, USA.

Address correspondence to: Dr. Chester C. Buckenmaier III, Department of Anesthesiology, Box 3094, Duke University Medical Center, Durham, North Carolina 27710, USA. Phone: 919-668-2053; Fax: 919-668-2081; E-mail: bucke001@mc.duke.edu

Financial support: Departmental.

Approved for publication January 29, 2002.

Revision approved March 13, 2002. 
described the use of peripheral nerve blocks in HOCM management. Given the paucity of information in the anesthesia literature, a review of the physiological implications of this rare but important disease process is useful.

Anesthetic goals for the HOCM patient have typically been directed towards achieving conditions that favour the hypertrophic heart, such as depressed myocardial contractility, normal or increased systemic vascular resistance (SVR), and increased left ventricular volume. ${ }^{4}$ Compared to general anesthesia, regional anesthesia techniques can provide superior postoperative analgesia, decreased surgical stress response and a reduction in postoperative nausea and vomiting which can benefit HOCM patients. Neuraxial anesthetic techniques however, are generally avoided in HOCM patients requiring thoracic surgery because of the sympathectomy and subsequent decrease in SVR frequently induced. We report the use of paravertebral blocks (PVB) in a HOCM patient who presented for partial mastectomy with axillary lymph node dissection. This report discusses the pertinent anesthetic treatment goals in HOCM patients and demonstrates how a novel peripheral nerve block may be used to satisfy these objectives.

\section{Case report}

A 72 -yr-old, $55 \mathrm{~kg}, 161 \mathrm{~cm}$, female, American Society of Anesthesiologists (ASA) class IV, presented for left partial mastectomy with axillary dissection for infiltrating ductal carcinoma. Her medical history was significant for intermittent shortness of breath for which she sought medical attention four years prior to presentation. She was diagnosed with non-small cell lung cancer and underwent a right upper lobectomy. HOCM was diagnosed by an echocardiogram performed following the lobectomy because of postoperative cardiac decompensation. She was noted to have a left ventricular outflow tract gradient of $69 \mathrm{mmHg}$ at that time. A persantine-thallium stress test was also performed, revealing mild anterior and inferior ischemia. Her medical history was also significant for hypertension, emphysema, tobacco use (30 pack/yr), and hyperlipidemia. She was maintained on home oxygen therapy $2 \mathrm{~L} \cdot \mathrm{min}^{-1}$ and peripheral oxygen saturation on room air was $88-90 \%$ noted on preadmission testing. Her HOCM was being managed successfully with daily oral verapamil $360 \mathrm{mg}$ and atenolol 100 $\mathrm{mg}$. Other medications included daily oral furosemide $40 \mathrm{mg}$, lisinopril $5 \mathrm{mg}$, atorvastatin $10 \mathrm{mg}$, potassium chloride $20 \mathrm{mg}$, and aspirin $100 \mathrm{mg}$.

The patient was counselled about options for anesthesia, including PVB. The patient agreed to this tech- nique with intraoperative sedation and conversion to general anesthesia if necessary. The possibility of an inadequate surgical block and the need for general anesthesia was considered preoperatively. Concurrence was reached with the patient's surgical team prior to the block concerning the necessity for escalation in patient monitoring should conversion to general anesthesia be required. Planned additional monitors included arterial blood pressure for direct blood pressure observations and access for arterial blood gas determinations. Central venous access would also be instituted to facilitate inotropic medication delivery and provide access for invasive cardiac monitoring if required. These monitors can guide medical therapy and facilitate rapid management of the hemodynamic instability that can occur with general anesthetics, particularly in HOCM patients. Since constant communication concerning the patient's well-being was possible with the PVB used in this case, these invasive monitors were not considered essential, but were immediately accessible. Finally, the availability (if needed) of postoperative management in a cardiac intensive care unit for this patient was established before beginning the procedure.

Standard ASA monitors were applied and supplemental oxygen at $10 \mathrm{~L} \cdot \mathrm{min}^{-1}$ was administered via facemask. Prior to PVB placement, external defibrillation pads were placed and connected to a defibrillator to facilitate treatment of an arrhythmia if it developed. The patient was given divided doses of $i v$ midazolam $2 \mathrm{mg}$ and fentanyl $100 \mu \mathrm{g}$ for moderate sedation during block placement. Her vital signs prior to block placement and after sedation were blood pressure $118 / 52 \mathrm{mmHg}$, heart rate 54 beats. $\mathrm{min}^{-1}$, and peripheral oxygen saturation of $100 \%$. The PVB were performed on the left side using the technique described by Greengrass and Steele ${ }^{5}$ with $5 \mathrm{~mL}$ of $0.5 \%$ ropivacaine and epinephrine $1: 400,000$ injected at thoracic levels $1-6$. The PVB were supplemented with a left superficial cervical plexus block using $5 \mathrm{~mL}$ of the same local anesthetic. This was done to block the supraclavicular nerves that provide sensation to the superior aspect of the breast. ${ }^{6}$

After demonstration of adequate blockade by loss of sensation to pinprick 15 min following block placement, the patient was transported to the operating room for surgery. Thirty minutes after completing the blocks the blood pressure decreased to $98 / 42 \mathrm{mmHg}$. This was treated with two doses of $i v$ phenylephrine $50 \mu \mathrm{g}$. There was no evidence of bilateral blockade or pneumothorax. This brief hypotension was attributed to the sedation administered during the block. Hypovolemia was another possibility because the patient had abstained from oral fluids for nearly $15 \mathrm{hr}$. 
After 6\% hetastarch infusion was begun the transient hypotension resolved and there were no further episodes of hypotension. She received a total of 700 $\mathrm{mL}$ of $6 \%$ hetastarch and $300 \mathrm{~mL}$ of lactated ringers during surgery. Prior to the first incision, midazolam $2 \mathrm{mg}$ and fentanyl $100 \mu \mathrm{g}$ (midazolam $4 \mathrm{mg}$ and fentanyl $200 \mu \mathrm{g}$ total used for the case) was titrated for sedation. No other medications were given. The patient remained comfortable and conversant during the two-hour procedure.

The partial mastectomy with left sentinel node biopsy was completed without complication and the patient was transferred to the postanesthesia care unit (PACU) for recovery. Her initial pain assessment in the PACU by verbal analogue scale (VAS, $0=$ no pain, $10=$ worst pain imaginable) was zero and she did not require any opioid medication until the following day. She received a total of $4 \mathrm{mg}$ of $i v$ morphine the following morning for a VAS of 2 and was subsequently discharged home pain free on postoperative day one. The patient was contacted by telephone four weeks later to discuss her home recovery. She stated she remained pain free after discharge from the hospital, did not require any further oral opioid medication for pain, and was extremely pleased with her anesthetic.

\section{Discussion}

We report the use of PVB in a patient with HOCM undergoing major surgery of the thorax. By administering local anesthetic near the somatic nerve roots unilateral anesthesia was provided without a profound bilateral sympathectomy. This technique facilitated maintenance of a normal hemodynamic status. The anesthetic provided adequate surgical conditions without the need for excessive opioid supplementation or sedation. Further, the PVB reduced the need for opioid pain medication after the operation until the following day, which is unusual for this type of surgery and essential in this debilitated patient because of the deleterious effect pain can have on cardiovascular function.

Compared to general anesthesia and thoracic epidural anesthesia, PVB offer several advantages for patients with HOCM. The genetic defect in HOCM results in generalized myocardial hypertrophy that tends to predominate in the interventricular septum leading to ventricular outflow obstruction. Outflow obstruction in HOCM often leads to massive hypertrophy of the left ventricle, which generates high left ventricular pressures that can compromise subendocardial blood flow. Sudden decreases in afterload or preload can accentuate the outflow obstruction leading to myocardial ischemia. The hypertrophied left ventricle also makes HOCM patients dependent on normal atrial function to preserve ventricular filling and maintain cardiac output. As a result cardiac arrhythmia can be particularly deleterious in HOCM patients. These changes place the HOCM patient at increased risk for life threatening ventricular outflow obstruction and myocardial ischemia when placed under surgical stress. Anesthetic goals for HOCM patients are directed at minimizing left ventricular outflow obstruction by decreasing myocardial contractility and increasing preload and afterload. ${ }^{7}$

Compared to general anesthesia, which would typically be employed in this patient, PVB can provide profound, long-lasting, sensory deafferentation. The resulting greater attenuation of the surgical stress response may translate into reduced inotropic stimulation of the heart. ${ }^{8}$ Additionally, unlike general anesthesia, PVB can provide superior postoperative analgesia and a reduction in postoperative nausea and vomiting. ${ }^{9,10}$ Regional anesthetic techniques such as spinal or epidural anesthesia, though efficacious in providing surgical anesthesia and sensory deafferentation, are often avoided because they produce bilateral sympathectomy. This may result in profound hypotension that can be difficult to treat in HOCM patients. Severe bradycardia following epidural anesthesia in an HOCM patient attributed to bilateral preganglionic sympathetic blockade has also been described. ${ }^{11}$

The incidence of hypotension with PVB is rare, though still of concern given the difficulty in resuscitating these patients. When choosing this regional anesthetic one must factor in the incidence of this problem compared to that likely to occur with more traditional techniques. In a series of 367 pediatric and adult patients, Lönnqvist et al. ${ }^{12}$ observed a $4.6 \%$ incidence of hypotension with this block. Hypotension following PVB can result from several causes. The paravertebral space is directly adjacent to the intervertebral foramina and as a result spread of local anesthetic into the epidural space or intrathecal space is possible. Contrast dye has also been demonstrated to pass from the paravertebral space, anterior to the vertebral bodies, to the contralateral side following a single level injection. ${ }^{13}$ These potential complications of PVB can be minimized by using lower local anesthetic volumes at each thoracic level and meticulous attention to technique when placing the blocks. The small volume $(5 \mathrm{~mL})$ used at each paravertebral level avoids a large neuraxial bolus and affords an extra margin of safety. Nevertheless, hypotension with PVB is still possible and should be treated aggressively.

The unilateral sympathectomy produced by PVB can unmask preexisting hypovolemia and result in hypotension. ${ }^{14}$ The brief episode of hypotension 
noted in this patient was most likely related to sedation during the block or possibly the unilateral sympathectomy and venous pooling following the PVB. Given the fact that this condition was rapidly treated with a colloid infusion demonstrates the sensitivity HOCM patients have to sympathectomy and need for rapid treatment.

Another potential complication of PVB is a pneumothorax. Anteriorly the paravertebral space is bordered by the pleura of the lung. Accidental needle penetration beyond the paravertebral space into the parenchyma of the lung is a consideration when employing PVB. The incidence of pneumothorax is rare when PVB is performed by an anesthesiologist experienced in the technique. ${ }^{12,14}$ However, the potential of a pneumothorax must still be considered carefully in HOCM patients, particularly when other comorbidities are involved.

The hemodynamic side effects inherent to most sedatives were avoided following the initial doses used for block placement. The minimal sedation enabled constant communication with the patient. This provided a valuable indicator of her physical well being during the procedure and avoided any other iatrogenic causes of hypotension.

This case demonstrates the pertinent anesthetic considerations in managing a patient with HOCM. In this patient the use of PVB resulted in stable hemodynamics, excellent unilateral analgesia, few side effects, and high patient satisfaction. PVB are a useful alternative anesthetic technique when faced with the HOCM patient requiring breast surgery.

\section{References}

1 Maron BJ, Gardin JM, Flack JM, Gidding SS, Kurosaki TT, Bild DE. Prevalence of hypertrophic cardiomyopathy in a general population of young adults. Echocardiographic analysis of 4111 subjects in the CARDIA study. Circulation 1995; 92: 785-9.

2 Cecchi F, Olivotto I, Montereggi A, Santoro G, Dolara A, Maron BJ. Hypertrophic cardiomyopathy in Tuscany: clinical course and outcome in an unselected regional population. J Am Coll Cardiol 1995; 26: 1529-36.

3 Haering JM, Comunale ME, Parker RA, et al. Cardiac risk of noncardiac surgery in patients with asymmetric septal hypertrophy. Anesthesiology 1996; 85: 254-9.

4 Thompson RC, Liberthson RR, Lowenstein E. Perioperative anesthetic risk of noncardiac surgery in hypertrophic obstructive cardiomyopathy. JAMA 1985; 254: 2419-21.

5 Greengrass $R$, Steele S. Paravertebral blocks for breast surgery. Techniques in Regional Anesth and Pain Man 1998; 2 : 8-12.
6 Brown DL. Cervical plexus block. In: Brown DL (Ed.). Regional Anesthesia and Analgesia, $2^{\text {nd }}$ ed. Philadelphia: W.B. Saunders Company, 1999: 181-5.

7 Stoelting RK, Dierdorf SF. Cardiomyopathies. In: Stoelting RK, Dierdorf SF (Eds.). Anesthesia and CoExisting Disease, 3rd ed. New York: Churchill Livingstone, 1993; 97-102.

8 Richardson J, Jones J, Atkinson R. The effect of thoracic paravertebral blockade on intercostal somatosensory evoked potentials. Anesth Analg 1998; 87: 373-6.

9 Klein SM, Bergh A, Steele SM, Georgiade GS, Greengrass $R A$. Thoracic paravertebral block for breast surgery. Anesth Analg 2000; 90: 1402-5.

10 Coveney E, Weltz CR, Greengrass $R$, et al. Use of paravertebral block anesthesia in the surgical management of breast cancer. Experience in 156 cases. Ann Surg 1998; 227: 496-501.

11 Baraka A, Jabbour S, Itani I. Severe bradycardia following epidural anesthesia in a patient with idiopathic hypertrophic subaortic stenosis (Letter). Anesth Analg 1987; 66: 1337-8.

12 Lönnqvist PA, MacKenzie J, Soni AK, Conacher ID. Paravertebral blockade. Failure rate and complications. Anaesthesia 1995; 50: 813-5.

13 Karmakar MK, Kwok WH, Kew J. Thoracic paravertebral block: radiological evidence of contralateral spread anterior to the vertebral bodies. Br J Anaesth 2000; 84: 263-5.

14 Karmakar MK. Thoracic paravertebral block. Anesthesiology 2001; 95: 771-80. 